\title{
Wanted: An International Jovian-Satellite-Phenomenon Centre
}

Niels $P$. Wieth-Knudsen

Svend Trostvej 12 IV, DK-1912 Fredriksberg C, Denmark

\begin{abstract}
Amateur observations made since 1977 show that although the ephemerides for the satellites of Jupiter published by the Jet Propulsion Laboratory are more accurate than those in the Astronomical Almanac, there are still small deviations, which may increase with time. Observation of eclipses, transits and occultations, as well as of the mutual phenomena that occur at 6-yearly intervals can provide information for correction of the ephemerides. There appear to be three groups coordinating observations: 1 ) in Germany, started by P. Ahnert in the early 1960 s and now under H.-J. Blasberg; 2) in the U.S.A., started by J. Ashbrook in 1976, and continued by J. Westfall of the ALPO; 3) in Australia and New Zealand, led by B. Loader. These groups appear to work independently, and it would greatly help research on this subject if there were an International Centre for collecting observations of the Jovian satellites - perhaps under the supervision of the IAU - whereby all observations made anywhere in the world would be available to anyone investigating the subject. Paris, where the predictions are calculated, might be a very suitable site for such a centre.
\end{abstract}

\section{The European Section of IOTA: Scientific Programme Coordination and Results}

W. Breisker and H.J. Bode

IOTA/ES, Bartold-Knaust-Str. 8, D-3000 Hannover 91, Fed. Rep. of Germany

\begin{abstract}
Lunar occultations and occultations of stars by planets and asteroids are important tools in determining astrometric information as well as details about the occulting bodies. Successful work requires a relatively large number of small observatories, well-distributed over a large area of the Earth. This is particularly important in occultations by asteroids because the accuracy of the predictions is generally poor.

Cooperation is therefore essential. IOTA/ES (European Section of the International Occultation Timing Association) provides a means of cooperative work, and holds meetings in different European countries about once a year. Eastern European countries are included.

Some results of occultations were presented and future events discussed. A relatively cheap photoelectric system was described, very suitable for amateurs and public observatories.
\end{abstract}

[Text not received. - Eds.] 